\title{
Integrating Agricultural Diversification in China's Major Policies for Sustainable and Resilient Crop Production
}

Thomas Wanger ( $\nabla$ tomcwanger@gmail.com )

Westlake University https://orcid.org/0000-0002-0435-1153

\section{Xueqing He}

Westlake University

\section{Wolfgang Weisser}

Technical University of Munich https://orcid.org/0000-0002-2757-8959

\section{Yi Zou}

Xi'an Jiaotong-Liverpool University https://orcid.org/0000-0002-7082-9258

\section{Shenggen Fan}

China Agricultural University https://orcid.org/0000-0002-2658-4863

\section{Thomas Crowther}

Swiss Federal Institute of Technology in Zurich https://orcid.org/0000-0001-5674-8913

Article

Keywords:

Posted Date: January 14th, 2022

DOI: https://doi.org/10.21203/rs.3.rs-1249283/v1

License: (1) This work is licensed under a Creative Commons Attribution 4.0 International License. Read Full License 


\section{Abstract}

Agricultural diversification of intensified farming systems is being proposed as a solution for achieving both food security and agricultural sustainability, but so far there has been little implementation of such policy at a larger scale. In China, major policies promote the "High-standard farmland consolidation" (HSFC) strategy to improve productivity and reduce environmental degradation in the world's largest food production areas by simplifying instead of diversifying landscapes on large instead of small fields. As China's Central government is asking for scientific innovations to improve its sustainable development strategy, we argue that China can become a role model to integrate agricultural diversification in its major policies, if HSFC builds on five decades of diversification research to achieve the national food security and sustainable development goals. We use text mining to analyze the past 17 years of China's most important agricultural policy, the No. 1 Central Documents (1CD) policy and show that agricultural diversification at the field and supply chain level has received limited attention. Based on global synthesis studies covering five decades of research, we provide practical recommendations of how to integrate agricultural diversification in the China's major policies from the national (the National 5-Year Plan and 1CD) to the provincial level. We use the major agricultural commodities rice, tea, wheat, and rapeseed in Zhejiang province as a case study to discuss how diversification can help to reach China's sustainable agriculture targets. Diversification of China's major food production areas on small fields could be an important example globally of how scientific progress informs policy and facilitates the food system transition.

\section{Full Text}

Agricultural diversification is being proposed as a solution for intensified agriculture to achieve both food security and agricultural sustainability by enhancing functional diversify at the field and landscape scale. Diversification practices such as crop rotation, agroforestry, and soil inoculation have been shown to enhance ecosystem services and protect biodiversity, with possible benefits for sustainable crop yields compared to conventional agriculture (1). Moreover, reducing the size of fields can increase crop yields and biodiversity that is critical for food production (2). Despite the increasing awareness for an agricultural paradigm shift and international efforts to enhance sustainable agricultural production (3), diversification is not yet implemented at a large scale. National agricultural policies are still failing to recognize the potential of agricultural diversification for reaching productivity goals in sustainable farming systems (4). However, a successful example of integrating agricultural diversification into major national policies would chart the path towards a global food system transition (5).

China is the perfect candidate to showcase the potential of agricultural diversification in the world's largest production systems for crops such as wheat, rice, potatoes, apples, and tobacco. Departing from a history of small but less productive farms, China has made significant advancements to reach food sovereignty. However, current and mechanized agricultural systems rely heavily on pesticide and fertilizer inputs that have caused environmental degradation and yield stagnations (6). Consequently, the Chinese government has passed strong policies to meet food security targets based on sustainable production, 
most notably the national 5-year-plan (N5YP; http://www.gov.cn/zhengce/2020-

11/03/content_5556991.htm) and the No. 1 Central Documents (1CD;

http://www.moa.gov.cn/ztzl/jj2021zyyhwj/). The recent $14^{\text {th }}$ N5YP emphasizes enhancing both food production capacity and sustainable agriculture. The $1 \mathrm{CD}$ is exclusively focused on national agriculture and aims to control agricultural pollution, stabilize yields, and increase farmers' income amongst other goals. To realize the key role of sustainable agriculture in these policies, China is pursuing its "highstandard farmland consolidation" (HSFC) strategy to simplify and operationalize the world's largest agricultural production areas in larger farms; Text S1). Current recommendations in HSFC focus on improving land productivity by increasing soil fertility, improving accessibility and irrigation, but seemly neglect diversification and farm size considerations, which may jeopardize China's sustainable food security targets. However, the government is actively inviting scientific innovations to improve its sustainable development strategies guided by N5YP, 1CD, and the provincial policies ( 7 ). This provides a unique opportunity to scale up agricultural diversification to reach agriculture-based sustainable development goals in China and the world.

Here, we argue that explicitly including both agricultural diversification and farm size considerations into Chinese major policies can turn HSFC into a major success story for China and set an example globally. First, we summarize the effects of agricultural diversification and farm size on biodiversity, ecosystem services, crop yields, and agricultural supply chains. We then use text mining tools on the past 17 years of $1 \mathrm{CD}$ policies to show that agricultural diversification has received limited attention, even within agricultural sustainability policies. We provide specific recommendations of how to integrate diversification into China's major policies from the national (5YNP, 1CD) to the provincial level and in the major agricultural commodities rice, tea, wheat, and rapeseed. We also recommend decreasing field sizes in the HSFC strategy to facilitate a food system transition in China's major agricultural production areas.

\section{Agriculture Diversification at multiple spatial levels and associated with the supply chain}

Five decades of international research on agricultural diversification and field size effects on yields and environment can now provide practical guidelines for sustainable agricultural development. At the regional level (several thousand square kilometers) at least $40 \%$ of forest should remain and be distributed at one and three quarters in a large and several small fragments, respectively (8). At the landscape and farm level, multiple diversification practices such as crop rotation, flower strips, and inoculation can have positive effects on farmers income, yield, biodiversity, and ecosystems services ( 1 , 9). Diversification can enhance carbon sequestration, nutrient cycling, soil fertility, water regulation, pollination, and pest control without compromising crop yields (1). In contrast, a reduction of landscape complexity reduces yield, $50 \%$ of which is due to agricultural biodiversity dependent biocontrol and pollination services (4). Maintaining $20 \%$ semi-natural habitat margin can ascertain a minimum level of biocontrol (4), which can help to reduce chemical inputs and, hence, pollution in agricultural lands. A global meta-analysis on the effect of field size on yields and agro-biodiversity found that smaller fields consistently enhance yields and biodiversity, suggesting that large fields cannot provide long-term sustainable food production (2). 
Along with the direct agricultural benefits, diversified fields and market access are important factors for increasing downstream benefits for the public's dietary diversity and nutrition including smallholder farmers (10). Market access can further increase cash incomes, allowing the purchasing more diverse foods from the market. In China, field-scale rice paddy diversification led to higher yields at lower pest loads and reduced pesticide use compared to non-diversified rice paddies (11). Hence, agricultural diversification offers yield, ecosystem service and biodiversity benefits that should be considered in China's major policies and HSFC.

Diversification associated with supply chains also provides opportunities for applied sustainable development innovations in technology, policy, and food market development, with far-reaching societal and economic consequences. Specifically, the post-farmgate supply chains are responsible for most economic activity in agricultural commodities and linked to changes in raw material and agricultural landscape properties. For example, it has been shown that the market channels, the agro-industrial and consumer demand, and the technological innovations for crop diversification with pea, linseed, and hemp must be established to process these crops in addition to the main crops (12). In addition, effective communication strategies must be implemented to connect all actors across the food supply chains to enable economic and sustainable innovations Consumer-driven mechanisms such as certification schemes that act along the supply chain can help to overcome barriers such as farmers' lack of knowledge, commitment, and financial incentives to adopt sustainable practices (13). Targeted financial investments can incentivize cooperation to support sustainable and diversified production strategies. Such policy and consumer driven strategies can be an important driver to advance supply chains associated with diversification. For China, advancing the topic of supply chains associated with diversifications provides important opportunities to improve access to market and, hence, income for millions of smallholder farmers.

\section{Opportunities of agricultural diversification in Chinese policies and farmland consolidation}

\section{Quantifying the importance of agricultural diversification in China's main agricultural policy.}

The No. 1 Central Document (1CD) is the first policy statement released by the central government each year since 2004, and is exclusively focused on national agriculture, rural areas, and farmer issues. We used text mining of all No. 1 Central Documents (1CD) from 2004 to 2021 (1CD for 2011 was excluded, because it is exclusively focusing on irrigation) to estimate the importance of agricultural diversification in these policies. We grouped diversification practices into eight categories according to $1 \mathrm{CD}$ content and Tamburini et al. (2020; Crop diversification, Supply chain associated with diversification, Inoculation, Noncrop diversification, Organic amendment, Organic farming, Integrated planting and animal breeding system, Reduced tillage). For details on the diversification practices and the text mining approach see Tab. S1 and Text S2.

Our results showed that diversification was a marginal topic in the past 17 years of the China's $1 \mathrm{CD}$ agricultural policies. The main aspect covered was "Diversification associated with supply chains" comprising $0.6 \%$ of the standardized word count of the 1 CD with peaks in peaks 2017 and 
2020 (Fig. 1), likely because increasing farmers' income has been a priority for the Chinese government. Agricultural diversification related to spatial scales remained low over time and was at most $0.2 \%$ of the standardized word count (Fig. 1). Specific recommendations for implementation largely concern organic amendments and integrated planting and animal breeding systems (Fig. 1). Overall, this shows that the strategy shift of the Chinese government from merely increasing the quantity of farmland before 2007 to incorporating ecosystem maintenance by 2010 and farmland restoration after 2016 (14) is not reflected in diversification strategies in the country's major agricultural policy. Sustainable agriculture as envisioned in the N5YP and the 1CD at the large scale is merely a local phenomenon on small areas (14).

\section{Towards practical and science-based policies through specific recommendations on diversification practices, farm sizes, and supply chains}

We follow China's call to propose scientific innovations to reach sustainable agricultural development goals by 2030 ( 7 ). We make specific recommendations for China's major national (N5YP and 1CD) and regional policies based on agricultural diversification, small field sizes, and when diversified raw materials are fully utilized in agricultural supply chains (Tab. 1).

The recent $14^{\text {th }}$ N5YP provides the broad development directions of China with an emphasis on enhanced food production capacity and sustainable agriculture. We suggest that China's N5YP should include and emphasize agricultural diversification at the landscape and field scale for sustainable agriculture development. Supply chain actors should enable diversified agricultural production through their readiness to process diversified raw materials and suitable technologies, by creating relevant markets and stimulating demand for certified sustainable products (Tab. 1).

As China's major and annual agricultural policy, the 1CD should continuously update diversification practices that can provide a practical guidance to reach the sustainable agricultural production goals in the $1 \mathrm{CD}$. Currently, there is limited discussion on how diversification strategies such as organic amendments, reduced tillage, crop diversification, non-crop diversification, inoculation, and organic farming can provide important opportunities to reach $1 \mathrm{CD}$ targets. It is critically important to consider how these diversification practices and a reduction of field size can potentially improve productivity and reduce chemical input in the HSFC strategy and in multifunctional farmland that connects the ecological, agricultural, and urban zones.

Provincial government policies are designed to help achieve 1CD goals through the HSFC strategy with the main crops and rural farm development in mind. Zhejiang province is an excellent example of how diversification can benefit China's agricultural sustainability and food security. First, the province has issued more than 30 policy documents related to the green development of agriculture, such as commercial organic fertilizer production and application, comprehensive straw utilization, and compensation mechanisms for ecological rice farming. Second, the province is actively building a green ecological agriculture policy system. Third, diversification practices are used in the province's main 
cropping systems including rice, tea, wheat, and rapeseed (15; Tab. 1, Tab. S2, Tab. S3). Fourth, the use of chemical fertilizers and pesticides has decreased seven years ahead of the country's goal to achieve zero growth of chemical fertilizer and pesticide application. Fifth, Zhejiang province is currently the only province on the list of National Agricultural Sustainable Development Experimental Demonstration Area, and it is amongst the first pilot areas of sustainable agricultural development. Hence, integrating diversification in Zhejiang's provincial policy can be a showcase for other provincial policies.

Agricultural supply chains should be set up to handle diversified raw materials and transfer the knowledge about the value of sustainable systems from the supply to the demand side. Technological innovations help supply chain actors to trace and process raw materials. Promoting sustainability standards and premium payments will ensure that producers adhere to sustainable strategies. Linking different business sectors from landscaping, tourism, and infrastructure in diversified landscapes provides a plethora of economic benefits ranging from rural job and income opportunities and avoided environmental externality costs. For detailed recommendations and how these will help to achieve 1CD targets, please refer to Tab. 1, Tab. S2, and Tab. S3.

Overall, it is important to note that our recommendations cannot be considered as final, but instead they reflect the current scientific thinking. Further research is always needed to refine this understanding, and the application of this knowledge should be considered in addition to - and not instead of - existing measures in China's major policies. Also, field sizes are traditionally smaller and the field size-productivity relationship in China are less well understood than in the EU and US (15). Thus, the general recommendation of field sizes of $<6$ ha may have to be scaled down to about $<4$ ha and adjusted based on the social and ecological context.

\section{Outlook}

Increasing farmers' income is one of the main themes in 1CD and farmers' income grew faster than urban residents' for 11 consecutive years since 2010, which support the causal link between policy text and actions. Scientific evidence suggests that China's major policies will only be able to achieve sustainable agricultural production through the national HSFC strategy if these policies include, and prioritize, agricultural diversification and a reduction in field sizes. The United Nations 2021 Food Systems Summit calls for such exemplary science-based policy changes to reach national sustainable development goals and to facilitate a global food system transition. Moreover, the UN's Decade on Ecosystem Restoration (2021-2030) recognizes farmlands as one of seven core ecosystems for restoration to reap benefits related to biodiversity conservation and carbon sequestration. On-farm restoration in HSFC through diversification will help to reach China's food security targets and at the same time the ambitious carbon reduction goals. Targeting diversified and optimally sized fields will also help to promote healthy diets, create markets, and hence, further improve smallholder income in China. Thus, integrating diversification into China's major policies to enhance sustainable farming on small fields will likely be an important example of how scientific progress informs policy and ultimately facilitates a food system transition globally. 


\section{Declarations}

Acknowledgments: We thank colleagues in the SAT lab and Jie Zeng for discussions on the analysis and an earlier version of this manuscript.

Funding: Westlake University startup grant to TWC

\section{Author contributions:}

Conceptualization: $\mathrm{XH}, \mathrm{TCW}$

Methodology: $\mathrm{XH}, \mathrm{TCW}$

Visualization: $\mathrm{XH}, \mathrm{TCW}$

Funding acquisition: TCW

Project administration: TCW

Supervision: TCW

Writing - original draft: $\mathrm{XH}, \mathrm{TCW}$

Writing - review \& editing: XH, TCW, YZ, WW, SF, TWC

Competing interests: Authors declare that they have no competing interests.

Data availability: All data is publicly available at www.gov.cn.

Code availability: The python code is available from the corresponding authors upon reasonable request.

\section{References}

1. G. Tamburini et al., Science Advances. 6, eaba1715 (2020).

2. V. Ricciardi, Z. Mehrabi, H. Wittman, D. James, N. Ramankutty, Nature Sustainability, 1-7 (2021).

3. T. C. Wanger et al., Nature Ecology \& Evolution. 4, 1150-1152 (2020).

4. T. Tscharntke, I. Grass, T. C. Wanger, C. Westphal, P. Batáry, Trends in Ecology \& Evolution. in press (2021).

5. W. Willett et al., The Lancet. 393, 447-492 (2019).

6. B. A. Bryan et al., Nature. 559, 193-204 (2018). 


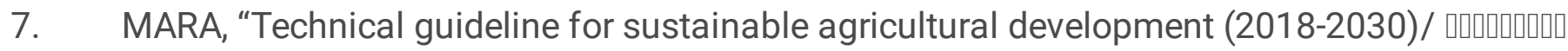

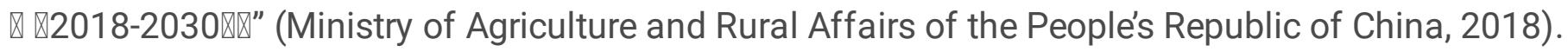

8. V. Arroyo-Rodríguez et al., Ecology Letters. 23, 1404-1420 (2020).

9. J. Rosa-Schleich, J. Loos, O. Mußhoff, T. Tscharntke, Ecological Economics. 160, 251-263 (2019).

10. J. Fanzo, D. Hunter, T. Borelli, F. Mattei, Diversifying food and diets: using agricultural biodiversity to improve nutrition and health (Routledge, 2013).

11. G. M. Gurr et al., Nature Plants. 2, 16014 (2016).

12. J.-M. Meynard et al., "Crop diversification: obstacles and levers. Study of farms and supply chains" (INRA, 2013), p. 59.

13. T. C. Wanger, L. W. Traill, R. Cooney, J. R. Rhodes, T. Tscharntke, Nature Ecology \& Evolution. 1, 1791-1793 (2017).

14. H. Tang, W. Yun, W. Liu, L. Sang, Land Use Policy. 89, 104212 (2019).

15. Y. Sheng, J. Ding, J. Huang, American Journal of Agricultural Economics. 101, 790-806 (2019).

\section{References Supplementary Information}

16. J. Huang, G. Yang. Global Food Security. 12, 119-26 (2017).

17. V. Arroyo-Rodríguez, et al. Ecology Letters. 23, 1404-20 (2020).

18. C. Sirami, et al. PNAS. 116, 16442-47 (2019).

19. E. Di-Clemente, J.M. Hernández-Mogollón, T. López-Guzmán, Soc. Sci. 9, 25 (2020).

20. S. K. Sachin, G. Angappa, A. G. Shradha. Internatinal Journal of Production Economics. 219, 179-194 (2020).

21. H. Guo, J. Bai, Y. Liu, J. Wang, J. Qu. China and Global Food Policy Report. AGFEP. 64-76 (2021)

22. Y. Qiao, et al. Ecological Economics. 145, 301-307 (2018).

23. Y. Zhang, et al. China and Global Food Policy Report. AGFEP. 14-30 (2021).

24. R. Bu, et al., Agriculture, Ecosystems \& Environment. 287, 106681(2020).

25. M. Liu, D. Zhang, W. Li. Chinese Journal of Eco-Agriculture. 18, 164-169 (2010).

26. B. Wen, et al. Agroforestry Systems. 94, 963-974 (2020). 
27. Z. Liu, Studies on nitrogen and phosphorus losses in surface runoff and their ecological integrated control in watershed of hilly tea field. Zhejiang University. (2012).

28. Y. Wang, et al.. Ying Yong Sheng Tai Xue Bao. 29, 3596囚3606 (2018).

29. X. Liu, Zhejiang Agriculture and Forest University. (2013)

30. D. Fu, et al. Journal of Integrated Agriculture. 15, 1673-168 (2016).

31. Z. Jin, et al. Science of the Total Environment. 649,1467-1480 (2019).

32. Z. Liu, et al. CATENA. 123, 45-51 (2014).

33. T. Lopes, et al. Pest Management Science. 72, 2193-2202 (2016).

34. L. Wang, et al. Agriculture Water Management. 229,105934 (2020).

\section{Table 1}

Table 1 is available in the Supplementary Files section.

\section{Figures}

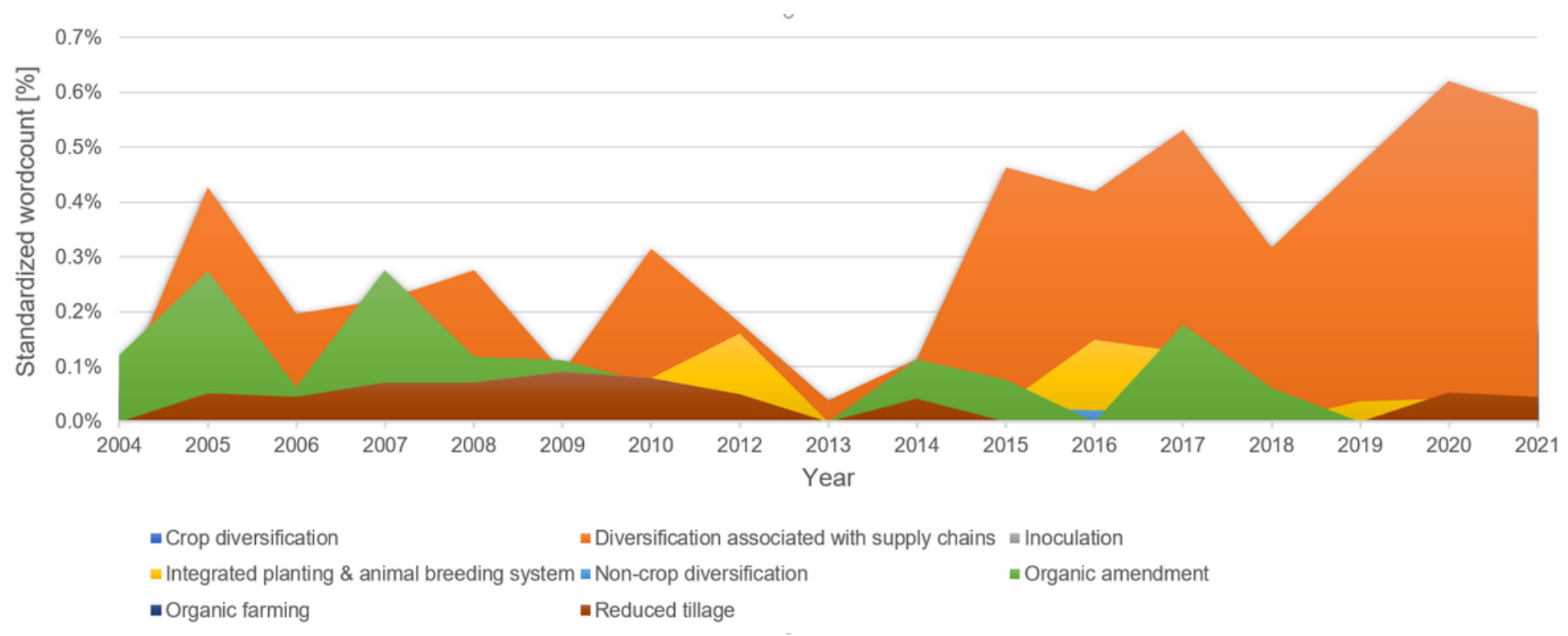

\section{Figure 1}

Relevance of spatial and supply chain diversification over 17 years of China's No.1 Central Document (1CD). Text mining showed that "supply chains associated with diversification" has been discussed to a limited extend in China's main agricultural policy. Agricultural diversification related to multiple spatial scales has been neglected. The data is standardized by the total number of words per policy. 


\section{Supplementary Files}

This is a list of supplementary files associated with this preprint. Click to download.

- SMHeNatureComms.docx

- floatimage2.png 